\title{
Association of Dyslipidemia and Glycated Haemoglobin in Gestational Diabetes Mellitus
}

\author{
M. Lenin ${ }^{1}$, R. Ramesh1* ${ }^{*}$, V. Kuzhandai Velu1, Seetesh Ghose ${ }^{2}$ \\ ${ }^{1}$ Department of Biochemistry, Mahatma Gandhi Medical College and Research Institute (SBVU), Pondicherry, India \\ ${ }^{2}$ Department of Obstetrics and Gynaecology, Mahatma Gandhi Medical College and Research Institute (SBVU), Pondicherry, \\ India \\ Email: *rameshrdr30@gmail.com
}

How to cite this paper: Lenin, M., Ramesh, R., Kuzhandai Velu, V. and Ghose, S. (2017) Association of Dyslipidemia and Glycated Haemoglobin in Gestational Diabetes Mellitus. Journal of Diabetes Mellitus, 7, 275-280.

https://doi.org/10.4236/jdm.2017.74022

Received: August 11, 2017

Accepted: October 23, 2017

Published: October 26, 2017

Copyright (C) 2017 by authors and Scientific Research Publishing Inc. This work is licensed under the Creative Commons Attribution International License (CC BY 4.0).

http://creativecommons.org/licenses/by/4.0/

Open Access

\begin{abstract}
Background: GDM is a condition where there is an onset of carbohydrate intolerance during pregnancy. In this condition many changes will take place in carbohydrate metabolism and other metabolic pathways especially in lipid metabolism. These metabolic changes associated with insulin resistance and dyslipidemia. We have aim to study the association between dyslipidemia and glycated haemoglobin. Materials and Methods: The study was conducted in 80 pregnant women of rural population of Puducherry in two groups. Group 1 includes 40 newly diagnosed GDM patients by DIPSI and Group II with 40 normal pregnant women, age group were between 20 to 40 years. Fasting blood glucose, 2-hour post glucose and lipid profile were estimated by auto analyzer. HbA1c was analysis by HPLC method. For comparisons of means student $\mathrm{t}$-test was used to determine the significance between GDM and controls. Results: There was statistically significant difference in lipid profiles $(F$ $=0.05), \mathrm{HDL}(P=0.04), \mathrm{VLDL}(P=0.00), \mathrm{LDL}(P=0.04) \mathrm{HDL}(P=0.04)$ in GDM groups. HbA1c $(P=0.02)$ levels were statistically significance with GDM pregnant women. There was no statically significant difference between FG $(P=0.23)$, TG $(P=0.30)$ and lipids profiles ratio levels in GDM. Conclusion: This study was found to be associations between lipids profiles and Gestational diabetes mellitus in GDM groups.
\end{abstract}

\section{Keywords}

Gestational Diabetes Mellitus, Lipid Profile, $\mathrm{HbA}_{1} \mathrm{c}$

\section{Introduction}

Gestational diabetes mellitus (GDM) is defined as carbohydrate intolerance with 
onset or first recognition during pregnancy [1]. Recent data shows $16.55 \%$ prevalence of GDM in our country and patient with GDM account for 90\% - 95\% of patients with diabetes during pregnancy women. GDM has an increased risk of developing other pregnancy related complications [2]. Pregnancy is a state of mild insulin resistance which is attributed to increased maternal adiposity and insulin desensitizing effects of placental products such as human placental lactogen, estrogen and prolactin, which is normally counter balanced with increased beta-cell proliferation and production of insulin [3]. Those patients who have limited beta cell capacity for the compensation of pregnancy induced insulin resistance due to variations in the genes involved in insulin secretion and utilization of carbohydrates is likely to develop GDM [4]. Diabetes and pregnancy have additive effect on the development of an atherogenic lipid profile. Lipid abnormalities are also called as diabetic dyslipidemia, which have been strongly associated with insulin resistance and lipid fractions, leading to elevated Triacylglycerol, LDL cholesterol and decrease level of HDL. LDL level alone was risk factor of cardiovascular diseases. In this present study we have to plan association of dyslipidemia and glycated haemoglobin.

\section{Materials and Methods}

The present case-control study was carried out at tertiary health care setup in Pondicherry after obtaining Institute Human Ethics committee approval and written informed consent from study subjects. In this study conducted in 80 pregnant women of rural population of Puducherry. 40 diagnosed GDM patients by DIPSI (The Diabetes in Pregnancy Study group India) and 40 normal pregnant women. Seventy five gm of anhydrous glucose was given to pregnant women irrespective of their fasting state. If after 2 hrs, blood glucose was $\geq 140$ $\mathrm{mg} / \mathrm{dl}$ then women were diagnosed as GDM. Age group of the study subjects were between 20 to 40 years. Approximately $3 \mathrm{ml}$ of fasting serum samples and 1 $\mathrm{ml}$ of whole blood samples will be collected in Clinical Chemistry laboratory for the estimation of fasting glucose (GOD-POD method), 2-hour post glucose levels and HbA1c (HPLC method), Lipids profile was estimated by IFCC approved clinical chemistry analyzer (fully automated). VLDL-C calculated by The Friedewald formula (FF) TG/5.

Statistics: Data was analyzed using statistical software package Epi Data V.2.2. Data were presented as mean $\pm \mathrm{SD}$. Comparisons of means student t-test was used to determine the significance between GDM and controls. Pearson correlation test were used for the association between $\mathrm{HbA1c}$ and VLDL, LDL markers.

\section{Result}

After appropriate screening, totally 40 GDM mother and equal number of normal pregnant women were included in this study. The comparison between the biochemical characteristic of the study groups is depicted in (Table 1). There 
Table 1. Mean and standard deviation of biochemical parameters in GDM.

\begin{tabular}{cccc}
\hline Parameters & Non GDM $($ Mean \pm SD $)$ & GDM $($ Mean \pm SD $)$ & ' $p^{\prime}$ value \\
\hline Weeks & $25 \pm 1.32$ & $25.26 \pm 1.4$ & 0.13 \\
Fasting glucose $(\mathrm{mg} / \mathrm{dL})$ & $72.45 \pm 7.23$ & $74.3 \pm 9.18$ & 0.23 \\
Age & $26.3 \pm 3.21$ & $27.2 \pm 4.1$ & 0.34 \\
BMI & $25.93 \pm 5.1$ & $25.4 \pm 4.8$ & 0.43 \\
DIPSI (mg/dL) & $161.68 \pm 11.02$ & $119.12 \pm 10.62$ & $0.00^{*}$ \\
HbAlc\% & $5.3 \pm 0.7$ & $6.7 \pm 1.2$ & $0.05^{\star}$ \\
Total Cholesterol mg/dL & $178.2 \pm 39.24$ & $142.2 \pm 31.2$ & $0.05^{\star}$ \\
Triacylglycrol mg/dL & $172.26 \pm 41.30$ & $182.24 \pm 38.45$ & 0.30 \\
HDL mg/dL & $72.81 \pm 24.67$ & $61.93 \pm 19.68$ & $0.04^{*}$ \\
LDL mg/dL & $77.33 \pm 37.9$ & $94.02 \pm 28.95$ & $0.04^{*}$ \\
VLDL & $34.8 \pm 8.18$ & $40.4 \pm 7.7$ & $0.00^{\star}$ \\
HDL/LDL ratio & $1.48 \pm 0.96$ & $1.52 \pm 0.87$ & 0.52 \\
TG/LDL ratio & $2.26 \pm 0.78$ & $2.54 \pm 0.91$ & 0.59 \\
TG/HDL ratio & $2.53 \pm 0.8$ & $3.6 \pm 1.2$ & 0.10 \\
TC/HDL ratio & $2.69 \pm 0.9$ & $3.17 \pm 1.0$ & 0.30 \\
\hline
\end{tabular}

${ }^{*} p<0.05$ statically significant

was statistically significant difference between total cholesterol levels $(p=0.05)$, Triacylglycrol $(p=0.02)$, LDL $(p=0.04), 2$-hour Post glucoselevels $(p=0.00)$ and also higher levels in GDM compare then control groups. HDL $(p=0.30)$ levels decrease in GDM compare then normal pregnancy groups but not statically significance. HbAlc $(p=0.02)$ levels were statistically significance with GDM pregnant women. There was no significant difference between GDM and lipids profiles ratio HDL/LDL $(p=0.52)$, TG/LDL $(p=0.59)$, TG/HDL $(p=0.10)$ and TC/HDL $(p=0.30)$ and BMI $(P=0.43)$.

Correlation analysis was done between $\mathrm{HbAlc}$ and lipid profile and its ration among GDM. The result suggest that HbAlc Vs LDL showed a positive correlation $(r=0.36)$ and HDL showed a negative correlation. Other parameters including lipid ratio didn't showed any correlation and results are present in Table 2 and Figure 1(a) \& Figure 1(b).

\section{Discussion}

Lipid profile and diabetes have been shown to be the important predictors for metabolic disturbances including dyslipidemia, hypertension, and cardiovascular diseases [7]. The abnormalities of Carbohydrate metabolism observed in GDM may lead to affect other metabolism also especially lipid metabolism [8]. Insulin resistance is one of the primary defects in the majority of patients with GDM, so this study was designed to observe the changes in lipid profile in normal pregnancy and GDM women [1]. 
Table 2. Correlation between glycemic status and lipids profiles. To find the association Pearson correlation analyzed was perforced between $\mathrm{HbAlc}$ and lipid profile in group 1 (GDM) HbAlc showed positive correlation with LDL $(r=0.36)$ and there was Negative correlation with HDL $(\mathrm{r}=-34)$. There was no correlate with among other markers.

\begin{tabular}{|c|c|c|c|}
\hline \multicolumn{2}{|c|}{ Biochemical Markers } & \multirow{2}{*}{$\begin{array}{l}\mathrm{r} \text { value } \\
0.488^{\star *}\end{array}$} & \multirow{2}{*}{$\frac{p \text { value }}{0.003}$} \\
\hline & Total Cholesterol & & \\
\hline & TGL & 0.072 & 0.685 \\
\hline & HDL & $-0.344^{\star}$ & 0.046 \\
\hline \multirow[t]{4}{*}{ HbAlc Vs } & LDL & 0.410 & 0.016 \\
\hline & TC/HDL & 0.193 & 0.275 \\
\hline & TGL/HDL & -0.076 & 0.670 \\
\hline & LDL/HDL & 0.233 & 0.184 \\
\hline
\end{tabular}

${ }^{*}$ Correlation is significant at the 0.01 level (2-tailed); ${ }^{\star}$ Correlation is significant at the 0.05 level (2-tailed).

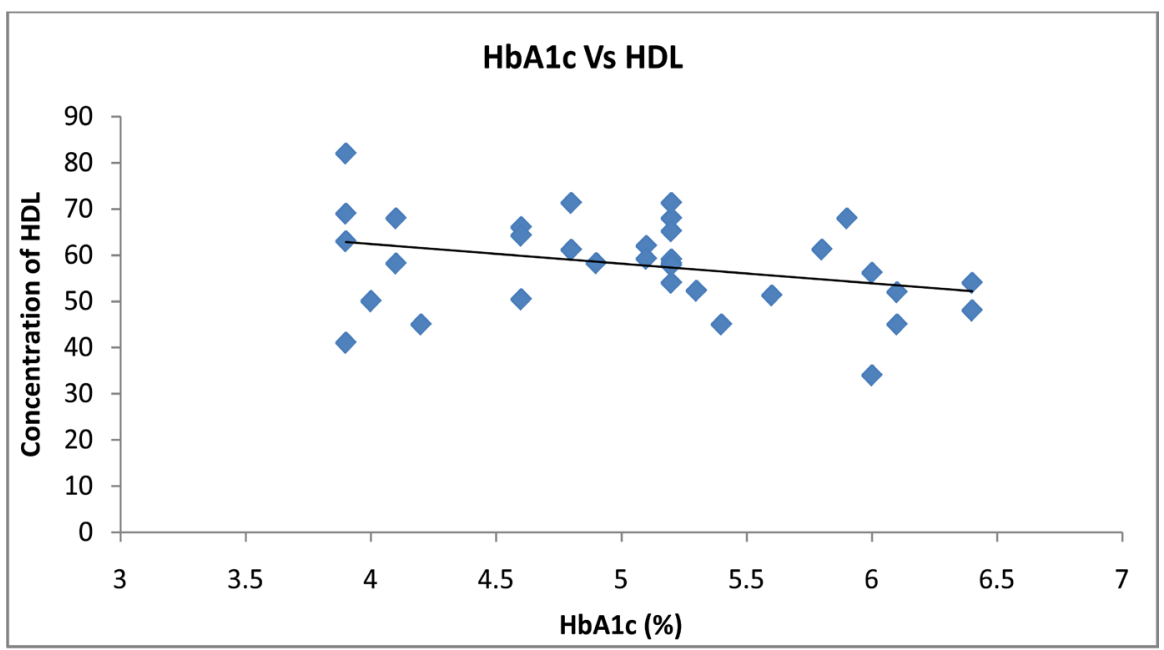

(a)

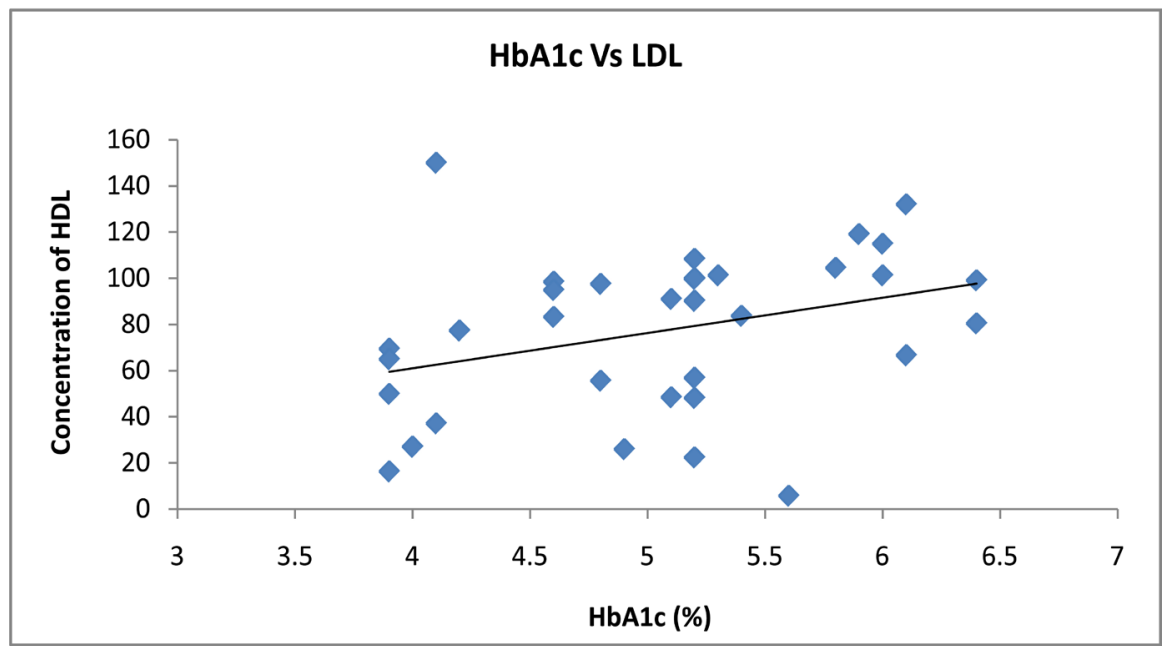

(b)

Figure 1. (a) Correlation between $\mathrm{HbA}_{1} \mathrm{c}$ Vs HDL; (b) correlation between $\mathrm{HbA}_{1} \mathrm{c} \mathrm{Vs}$ LDL. 
This present study has shown that TC $(p=0.05)$, LDL $(p=0.04)$, LDL $(p=$ $0.04)$, VLDL $(p=0.00)$, these lipid profiles are higher and statistically significance in GDM groups. HDL $(p=0.04)$ levels was little decrease in GDM groups compare then control groups and also statistically significance with GDM groups. According Fredrick et al., Michel et al in type 2 diabetes mellitus there is significance elevation of TC and decreasing HDL. Our study has shown similar results except for TC, LDL significantly elevated.There was no statistical difference in HDL/LDL ratio $(p=0.50)$, TG/LDL ratio $(p=0.59)$, TG/HDL $(p=0.10)$, TG/HDL (0.3) ratio in patients with GDM and normal Pregnant women. These finding are in accordance with the studies of kalpana et al., these lipids profiles ratio little increase because due to increased oxidation of free fatty acids lead to increased concentrations of acetyl CoA which will exceeds to cause to TCA cycle in liver Acetyl CoA is regulated to synthesis of cholesterol, fatty acids and triglycerides [9].

Our study had shown highly correlation between hbAlc and lipids profiles in GDM groups [10] similar result observed by Samatha et al. shows significance correlation between hbAlc and various lipids profiles Barbara V. Howard et al. 1984; James S. Reitman et al. but some authors, Baker et al., have observed variant observation where there was no correlation between the two groups. Schaefer-Graf et al. documented that maternal serum triglyceride levels significantly correlated with abnormal fetal growth in women with gestational diabetes mellitus [11] [12]. Which predominately increase levels of triglyceride production, free fatty acids and glucose.

In our study shows TG levels statistically higher in GDM groups compare then control groups. In Insulin resistance condition and type 2 diabetes mellitus are associated with a clustering of interrelated lipids metabolism abnormalities, which include elevated triglyceride concentrations, decreased HDL cholesterol and a predominance of LDL-cholesterol particles [13]. In addition, Bartha and colleagues reported that maternal total cholesterol and triglyceride concentrations were similar in GDM and normal pregnancy. Our study shows dyslipidemia in gestational diabetes mellitus. Most of studies were shown an $\mathrm{HbAlc}$ level was statically significance with GDM groups. In present study report HbAlc $(P=0.02)$ also shown statistically significance with GDM. In Diabetes patients most predictive marker is HbAlc compare then fasting glucose levels and post prandial glucose levels [14]. C. Capula et al., shown HbAlc levels a good predictor of adverse pregnancy outcomes. The limitation of the study was sample size was low and have to analyze for insulin resistance markers in Gestational diabetes mellitus.

\section{Conclusion}

This study found that GDM is associated with lipids profiles in GDM groups. Early diagnosis of abnormalities of lipids profiles reduced to severity of maternal and fetal complications. It would be helpful for the early detection and treatment of GDM groups. 


\section{References}

[1] Gilmartin, A. "Bird” H., Ural, S.H. and Repke, J.T. (2008) Gestational Diabetes Mellitus. Reviews in Obstetrics and Gynecology, 1, 129-134.

[2] Yasmin, T. (2012) Iffat Fatima with Saima Yasmin Qadir. Maternal and Foetal Outcome in Gestational Diabetes. JAMC, 24, 3-4.

[3] Ryan, E.A. and Enns, L. (1988) Role of Gestational Hormones in the Induction of Insulin Resistance. The Journal of Clinical Endocrinology \& Metabolism, 67, 341-347. https://doi.org/10.1210/jcem-67-2-341

[4] Sonagra, A.D., Biradar, S.M., K. D. and Murthy, D.S.J. (2014) Normal Pregnancy-A State of Insulin Resistance. Journal of Clinical and Diagnostic Research: $J C D R, 8, \mathrm{CC} 01-\mathrm{CC} 03$.

[5] Wilcox, G. (2005) Insulin and Insulin Resistance. The Clinical Biochemist Reviews, 26, 19-39.

[6] Basa, A.L.P. and Garber, A.J. (2001) Cardiovascular Disease and Diabetes: Modifying Risk Factors Other Than Glucose Control. The Ochsner Journal, 3, 132-137.

[7] Ozder, A. (2014) Lipid Profile Abnormalities Seen in T2DM Patients in Primary Healthcare in Turkey: A Cross-Sectional Study. Lipids in Health and Disease [Internet]. http://www.ncbi.nlm.nih.gov/pmc/articles/PMC4271485/

[8] American Diabetes Association. Diagnosis and Classification of Diabetes Mellitus. 31.

[9] Horton, J.D., Shimomura, I., Brown, M.S., Hammer, R.E., Goldstein, J.L. and Shimano, H. (1998) Activation of Cholesterol Synthesis in Preference to Fatty Acid Synthesis in Liver and Adipose Tissue of Transgenic Mice Overproducing Sterol Regulatory Element-Binding Protein-2. Journal of Clinical Investigation, 101, 2331-2339. https://doi.org/10.1172/JCI2961

[10] Eslamian, L., Akbari, S., Marsoosi, V. and Jamal, A. (2013) Effect of Different Maternal Metabolic Characteristics on Fetal Growth in Women with Gestational Diabetes Mellitus. International Journal of Reproductive BioMedicine, 11, 325-334.

[11] Vani, K. (2015) Alterations in Lipid Profile in Gestational Diabetes Mellitus (GDM) and Type 2 DM Women during Pregnancy. International Journal of Medical Research and Review [Internet] http://medresearch.in/index.php/IJMRR/article/view/351

[12] Bissonnette, S., Salem, H., Wassef, H., Saint-Pierre, N., Tardif, A., Baass, A., et al. (2013) Low Density Lipoprotein Delays Clearance of Triglyceride-Rich Lipoprotein by Human Subcutaneous Adipose Tissue. The Journal of Lipid Research, 54, 1466-1476. https://doi.org/10.1194/jlr.P023176

[13] Han, E.S., Krauss, R.M., Xu, F., Sridhar, S.B., Ferrara, A., Quesenberry, C.P., et al. (2016) Prepregnancy Adverse Lipid Profile and Subsequent Risk of Gestational Diabetes. The Journal of Clinical Endocrinology \& Metabolism, 101, 2721-2727. https://doi.org/10.1210/jc.2015-3904

[14] Ghazanfari, Z., Haghdoost, A.A., Alizadeh, S.M., Atapour, J. and Zolala, F. (2010) A Comparison of HbAlc and Fasting Blood Sugar Tests in General Population. International Journal of Preventive Medicine, 1, 187-194. 